\title{
Análise da evolução de esporotricose empregando modelo de regressão em casos de felinos de Timbaúba/PE - Brasil
}

Analysis of sporotricose evolution using regression model in cases of felines from Timbaúlba / PE Brazil

Análisis de la evolución de sporotricosa mediante modelo de regresión en casos de felinas de Timbaúba / PE - Brasil

José Eduardo Silva

ORCID: https://orcid.org/0000-0002-8483-0305 Universidade Federal Rural de Pernambuco, Brasil E-mail: profeduardosilva3@gmail.com

Marcondes Domingos de Araújo Cirino ORCID: https://orcid.org/0000-0002-5293-0213 Universidade Federal de Campina Grande, Brasil E-mail: marcondomingos@gmail.com

Elaine Fonseca de Farias ORCID: https://orcid.org/0000-0003-0162-1186 Universidade Federal Rural de Pernambuco, Brasil E-mail:elainefarias_vet@hotmail.com Larissa Ferreira da Fonseca

ORCID: https://orcid.org/0000-0002-1763-5833 Universidade de Pernambuco, Brasil E-mail: Larissa.f.fonsec@gmail.com

Gisele Mine Shinohara

ORCID: https://orcid.org/0000-0003-4068-5764 Universidade Federal de Pernambuco, Brasil E-mail: giseleshinohara96@gmail.com

Neide Kazue Sakugawa Shinohara ORCID: https://orcid.org/0000-0001-8356-874X Universidade Federal Rural de Pernambuco, Brasil E-mail: neideshinohara@gmail.com

Moacyr Cunha Filho

ORCID: https://orcid.org/0000-0002-3466-8143 Universidade Federal Rural de Pernambuco, Brasil E-mail: moacyr2006@gmail.com

\begin{abstract}
Resumo
Objetivo: analisar a evolução de casos confirmados de esporotricose no município de Timbaúba - PE com o uso de Modelos de Regressão e Previsão. Método: foram utilizados dados mensais de casos confirmados de esporotricose por meio de testes clínicos e laboratorial, fornecidos por uma Unidade de Saúde Animal, do período de janeiro a dezembro de 2020. Com os dados foram realizados testes estatísticos com os Modelos de Regressão: linear simples, cúbico e quadrático. Além de testes de previsão com o Modelo de Média Simples. Resultados: as análises o Modelo de Regressão Cúbico demonstrou o melhor ajuste para o conjunto de dados de casos confirmados de esporotricose no município. Conclusão: com a análise foi possível perceber a oscilação em torno de 3 casos confirmados, sem tendência de mudança, cuja previsão para o mês de janeiro de 2021 reside na evidência de ocorrência de 2,66 casos à 95\% de confiança.
\end{abstract}

Palavras-chave: Modelo matemático; Sporothrix schenckii; Média móvel; Teste de previsão.

\begin{abstract}
Objective: to analyze the evolution of confirmed cases of sporotrichosis in the municipality of Timbaúba - PE using Regression and Prediction Models. Method: monthly data of confirmed cases of sporotrichosis were used through clinical and laboratory tests, provided by an Animal Health Unit, from January to December 2020. With the data, statistical tests were performed with the Regression Models: linear simple, cubic and quadratic. In addition to prediction tests with the Simple Average Model. Results: the analysis of the Cubic Regression Model demonstrated the best fit for the data set of confirmed cases of sporotrichosis in the municipality. Conclusion: with the analysis it
\end{abstract}


was possible to perceive the oscillation around 3 confirmed cases, with no trend of change, whose forecast for the month of January 2021 lies in the evidence of the occurrence of 2.66 cases at $95 \%$ confidence.

Keywords: Mathematical model; Sporothrix schenckii; Moving average; Prediction test.

\section{Resumen}

Objetivo: analizar la evolución de casos confirmados de esporotricosis en el municipio de Timbaúba - PE mediante modelos de regresión y predicción. Método: se utilizaron datos mensuales de casos confirmados de esporotricosis mediante pruebas clínicas y de laboratorio, provistas por una Unidad de Sanidad Animal, de enero a diciembre de 2020. Con los datos se realizaron pruebas estadísticas con los Modelos de Regresión: lineal simple, cúbica y cuadrática. Además de las pruebas de predicción con el Modelo de Promedio Simple. Resultados: el análisis del Modelo de Regresión Cúbica demostró el mejor ajuste para el conjunto de datos de casos confirmados de esporotricosis en el municipio. Conclusión: con el análisis se pudo percibir la oscilación en torno a 3 casos confirmados, sin tendencia de cambio, cuya previsión para el mes de enero de 2021 radica en la evidencia de la ocurrencia de 2,66 casos al $95 \%$ de confianza.

Palabras clave: Modelo matemático; Sporothrix schenckii; Media móvil; Prueba de predicción.

\section{Introdução}

Esporotricose é uma afecção fúngica subcutânea granulomatosa que pode se disseminar sistemicamente para outros órgãos, principalmente pulmões e fígado de gatos, cachorros e humanos, considerada como uma micose de implantação (Bittencourt et al., 2016). Sua etiologia é atribuída principalmente ao complexo Sporothrix schenckii (Araujo \& Leal, 2016; Sizar \& Talati, 2019). O habitat natural é em troncos de árvore, material terroso e outros materiais orgânicos contaminados com o fungo. Devido ao hábito de arranhadura e de cavar a terra pelos felinos, ocorre o acúmulo do fungo em suas unhas, tornando-se importantes transmissores dessa infecção fúngica (Rios, Suarez, Moreno \& Moreno, 2018).

A via de transmissão mais frequente é através de mordida ou arranhadura de animais infectados na pele ou na mucosa do hospedeiro, que pode ser outro animal ou o humano, sendo a via respiratória menos frequente. Nos felinos, desenvolve-se no local da inoculação do fungo, uma pápula ou nódulo cutâneo que posteriormente, evolui para uma úlcera e pode se disseminar com múltiplas lesões na pele, associado frequentemente a sintomas respiratórios nos felinos (de Souza et al., 2018). Há uma diferença entre a proliferação de organismos nas espécies, nos gatos ocorre uma produção elevada desses organismos em contrapartida nos cães a ocorrência do agente na avaliação citológica é menor e demanda uma avaliação bastante acurada (Cowell, Tyler, Meinkoth \& DeNicola, 2009).

Apesar de ser uma doença mais incidente nos países tropicais e subtropicais do continente americano, o Sporothrix schenckii possui distribuição mundial. No Brasil, durante os últimos anos, houve um crescimento relevante de casos de esporotricose, com maior epidemiologia em felinos, apresentando ferimentos e úlcera cutânea (Mascarenhas et al., 2019; Silva et al., 2020a).

Desta forma, a esporotricose por ser uma zoonose necessita de estudos mais aprofundados que evidenciem os surtos epidêmicos em diversas regiões brasileiras, bem como estudos acerca de evolução e tratamento que subsidiem métodos e políticas públicas no combate a desinformação, cuidados e tratamentos.

Considerando a perspectiva zoonótica existem diversos estudos e pesquisas em andamento que utilizam modelos matemáticos para entender a evolução de surtos epidêmicos, como as viroses Zika e Covid-19. A Epidemiologia Matemática contribui com uma série de modelos que recentemente vem sendo utilizados nos estudos da pandemia do Covid-19, dentre eles: modelos de regressão não lineares (do Amaral et al., 2020; dos Santos et al., 2020). Possuindo as características de transmissibilidade e mobilidade, um modelo matemático representa um sistema de dados e informações relevantes com potencial condição de mutação contemplando diversas áreas do conhecimento (biologia, estatística, matemática).

Assim, os felinos acometidos por esse fungo que estão próximos aos humanos se constituem vetor biológico na implantação traumática do fungo no tecido subcutâneo humano, através do hábito de arranhar e lamber (de Macêdo-Sales et al., 2018). Portanto, como a esporotricose é uma patologia de transmissão zoonótica através do felino doméstico (Felis catus), 
subnotificada e negligenciada na saúde pública, o artigo teve como objetivo analisar a evolução de casos confirmados de esporotricose no município de Timbaúba - PE com o uso de Modelos de Regressão e de Previsão.

\section{Metodologia}

\subsection{Descrição dos dados}

Nesta pesquisa os autores (as) utilizaram dados coletados de procedimentos realizado em atendimento, diagnóstico e tratamento da esporotricose, obtidos no Centro Veterinário Santa Cândida, no Município de Timbaúba, no período de janeiro a dezembro de 2020, totalizando 215 animais atendidos, deste total 37 animais testaram positivo para esporotricose e foram submetidos e acompanhados com tratamentos terapêuticos.

Neste sentido, a pesquisa se enquadra em um método quantitativo pela possibilidade viável de elucidações científicas com base em modelos matemáticos (Pereira et al., 2018), na Figura 1 consta a localização geográfica da cidade de Timbaúba PE.

Figura 1. Mapa de localização geográfica do local de estudo.

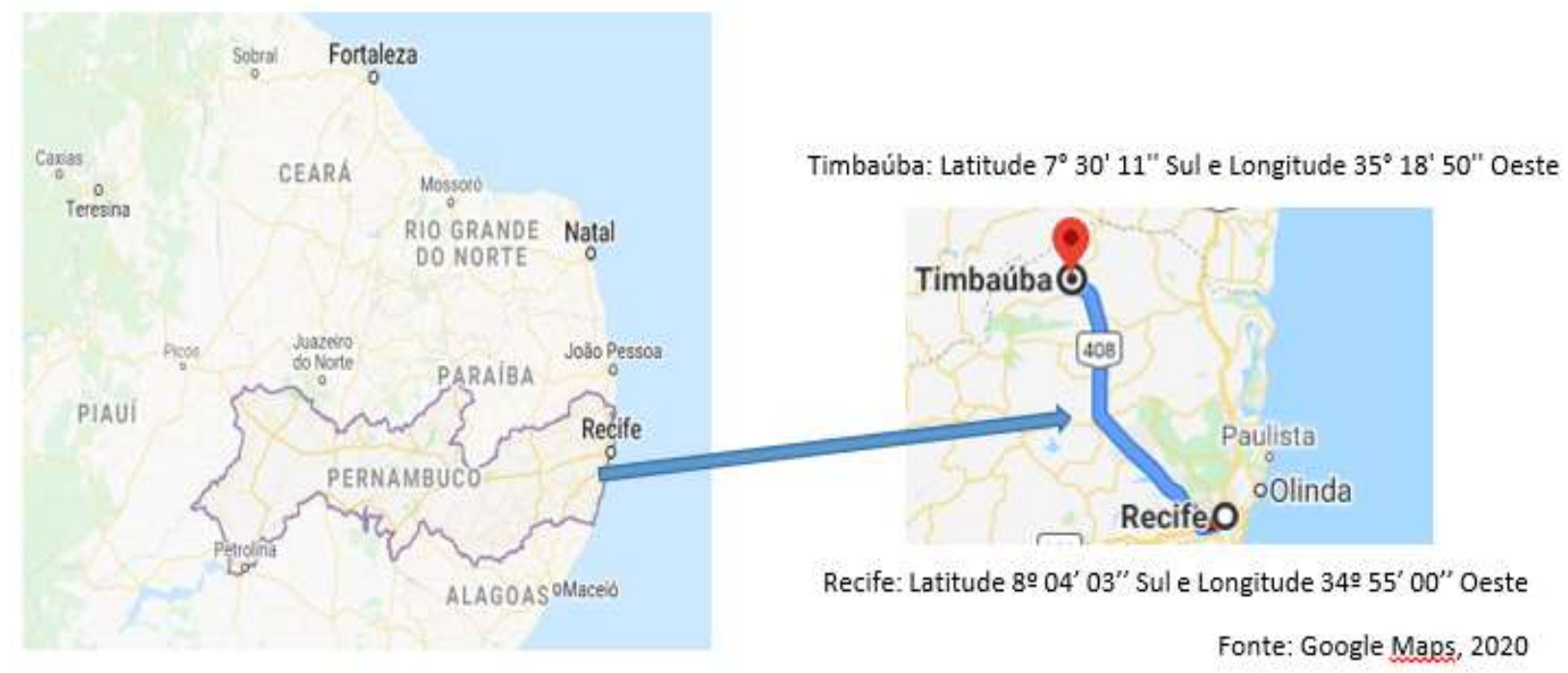

O município de Timbaúba está localizado na Zona da Mata Norte do Estado de Pernambuco, região Nordeste do Brasil, abrange os distritos de Cruangi, Livramento do Tiúma, Catucá e Queimadas. Sua posição geográfica reside na unidade geoambiental do Planalto da Borborema.

Na Tabela 1, observamos os dados coletados com os registros de atendimentos, casos confirmados positivos e negativas, bem como em tratamento. 
Tabela 1. Descrição dos Dados Coletados sobre casos de esporotricose.

\begin{tabular}{|c|c|c|c|c|}
\hline \multicolumn{5}{|c|}{ APRESENTAÇÃO DOS DADOS (2020) } \\
\hline PERÍODO & Atendimentos & Teste positivo & Teste negativo & Em tratamento \\
\hline JANEIRO & 14 & 2 & 1 & 2 \\
\hline FEVEREIRO & 18 & 6 & 2 & 9 \\
\hline MARÇO & 22 & 3 & 0 & 3 \\
\hline ABRIL & 16 & 3 & 1 & 3 \\
\hline MAIO & 19 & 1 & 0 & 1 \\
\hline JUNHO & 18 & 3 & 1 & 3 \\
\hline JULHO & 17 & 2 & 0 & 2 \\
\hline AGOSTO & 19 & 1 & 0 & 1 \\
\hline SETEMBRO & 22 & 8 & 1 & 8 \\
\hline OUTUBRO & 18 & 2 & 1 & 2 \\
\hline NOVEMBRO & 19 & 5 & 0 & 5 \\
\hline DEZEMBRO & 13 & 1 & 1 & 1 \\
\hline \multicolumn{5}{|c|}{ TOTAIS } \\
\hline 12 & 215 & 37 & 08 & 37 \\
\hline MÉDIA & 17,91 & 3,08 & 0,66 & 3,08 \\
\hline
\end{tabular}

Fonte: Centro Veterinário Santa Cândida - Município de Timbaúba.

Conforme a Tabela 1 dos 215 animais atendidos no ano de 2020, apenas 45 deles tiveram suspeita clínica para esporotricose e ao serem submetidos aos testes laboratoriais 37 resultaram positivo para doença e encaminhados para tratamento.

\subsection{Materiais e Métodos}

Neste estudo optou-se por utilizar Modelos de Regressão (Canhanga, 2020) para verificar o comportamento da evolução de casos de esporotricose. Foram selecionados os Modelos: Modelo Linear Simples, Modelo Quadrático e Modelo Cúbico (Souza, 2016; do Amaral et al., 2020). Na Tabela 2, estão as respectivas equações estatísticas de cada modelo.

Tabela 2. Descrição das equações estatísticas.

\begin{tabular}{l|c}
\hline \multicolumn{1}{c|}{ Modelo de Regressão } & Equação \\
\hline Linear Simples & $Y_{i}=\beta_{0}+\beta_{1} X_{i}+\epsilon_{i}$, para $\mathrm{i}=1, \ldots, \mathrm{n}$ \\
Quadrático & $Y_{i}=\beta_{0}+\beta_{1} X_{i}+\beta_{2} X_{i}^{2}+\epsilon_{i}$, para $\mathrm{i}=1, \ldots, \mathrm{n}$ \\
Cúbico & $Y_{i}=\beta_{0}+\beta_{1} X_{i}+\beta_{2} X_{i}^{2}+\beta_{3} X_{i}^{3}+\epsilon_{i}$, para i=1, .., n \\
& \\
\hline
\end{tabular}

Fonte: Autores (2020).

Posteriormente foram obtidas as estimativas dos parâmetros de cada modelo utilizando como critério para seleção do melhor modelo, o Coeficiente de Determinação $\left(R^{2}\right)$ (Silva et al., 2020b) que é dado pela equação:

$$
R^{2}=\frac{S Q R}{S Q T}=1-\frac{S Q E}{S Q T}=\frac{\hat{\beta}_{1} \sum_{i=1}^{n}\left(x_{i}-\bar{x}\right) Y_{i}}{\sum_{i=1}^{n}\left(Y_{i}-\bar{Y}\right)^{2}}
$$

Com os dados obtidos, observa-se uma série temporal cuja variável observada dispõe uma sequência no decorrer do tempo (Morettin \& Toloi, 2006). Diante disto obtém-se condições par realização de previsões de possíveis acontecimentos futuros (Camelo, Lucio, Leal Junior \& Carvalho, 2017). 
Prever tendência e sazonalidade com dados em uma série temporal pressupõe que o pesquisador escolha adequadamente um modelo para inferir estatisticamente sobre os dados, como utilizar o modelo aditivo cujos efeitos são diferenciados ou modelo multiplicativo cuja ideia de que à medida que os dados aumentam o padrão de sazonalidade também aumenta (Morettin \& Toloi, 2006).

Neste sentido os métodos para estimação de uma tendência podem ser por ajuste de uma função de tempo, suavização dos valores da série em torno de um ponto, ou ainda, suavização da série por meio de sucessivos ajustes de retas.

O uso do Modelo de Média Móvel verifica a previsão de novos casos de esporotricose no município de Timbaúba PE, esse modelo permite a utilização de médias baseadas em dados passados de uma série temporal, para previsão futura das médias de observações passadas (Couto, Oliveira \& Braga, 2016), utilizando como suporte ao critério de decisão do modelo a acurácia de comprimento para o Desvio Padrão Absoluto da Média (MAD) e Desvio Padrão Quadrático da Média (MSD). Neste sentido, foi utilizado o software Action Stat $®$, versão 3.7 de teste para realizar todas as análises.

Quanto ao diagnóstico e tratamento, existem várias técnicas para coleta do material para análise laboratorial. A técnica Biópsia por Agulha Fina (BAF) pode ser empregada de duas formas: a primeira forma com a agulha acoplada a seringa onde ocorre a introdução da agulha na lesão e o êmbolo é tracionado exercendo assim uma pressão negativa, é feito o movimento de "vai e vem" sem que a agulha seja completamente removida e então a pressão negativa é desfeita. O material coletado é depositado na lâmina de microscopia.

A segunda forma utiliza apenas a agulha não sendo utilizado a seringa para a pressão negativa, está técnica também é conhecida como técnica de capilaridade, consiste em na introdução da agulha na lesão e com os movimentos de "vai e vem" o material é coletado, por fim uma seringa contendo ar é acoplada a agulha e o material é ejetado em uma lâmina de microscopia. Pode-se também utilizar a técnica de impressão, onde a lâmina de vidro é posta diretamente em contato com a lesão do animal para coleta do material (Cowell, Tyler, Meinkoth \& DeNicola, 2009).

\section{Resultados e Discussão}

Utilizamos para efeito das análises a média móvel de quatro meses (Figura 2), para avaliação dos modelos propostos, onde os Coeficientes de Determinação $\left(R^{2}\right)$ são apresentados na Tabela 2.

Figura 2. Média Móvel de janela 4.

\section{Média móvel}

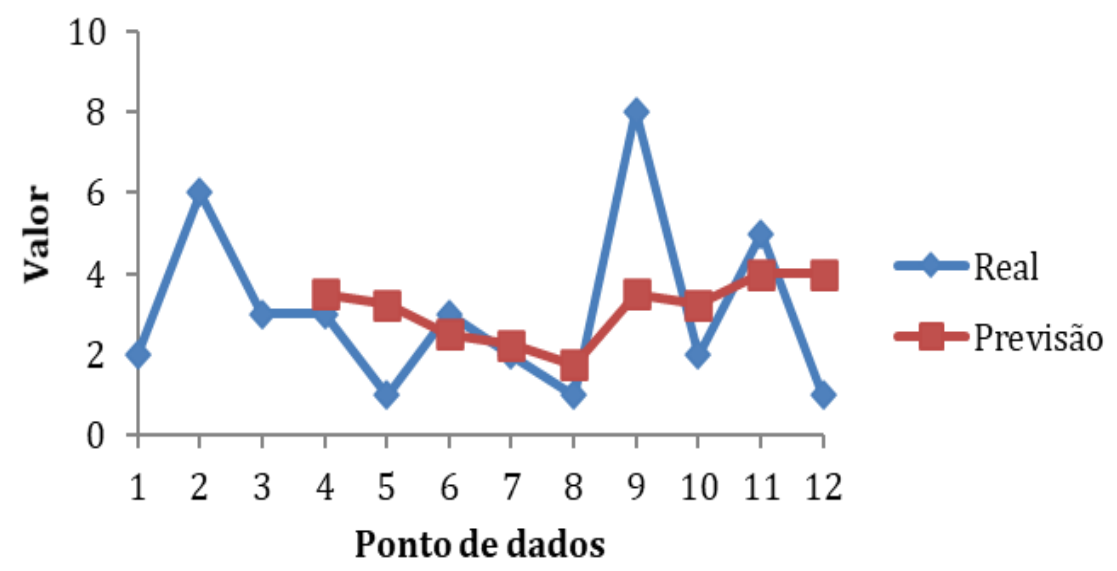

Fonte: Autores (2020). 
Tabela 2. Descrição dos Coeficientes de Determinação.

\begin{tabular}{l|l}
\hline \multicolumn{1}{c|}{ Modelo de Regressão } & $\left(R^{2}\right)$ \\
\hline Linear Simples & 0,16 \\
Quadrático & 0,66 \\
Cúbico & 0,74 \\
\hline
\end{tabular}

Fonte: Autores (2020).

Observa-se o Coeficiente de Determinação $\left(R^{2}\right)$, contido na Tabela 2, indicando que o Modelo Cúbico apresentou cerca de $74 \%$ da variância total explicada pela regressão, com este resultado o Modelo Cúbico se apresenta como o modelo que melhor se ajustou aos dados. Sendo assim, os pressupostos de normalidade, homoscedasticidade e independência são atendidos pelo modelo. Pela análise gráfica o Teste de Shapiro-Wilk demonstrou que os dados seguem normalidade $(\mathrm{p}$-valor $=0,84)$, conforme Figura 3.

Figura 3. Análise Gráfica da Distribuição dos Dados.

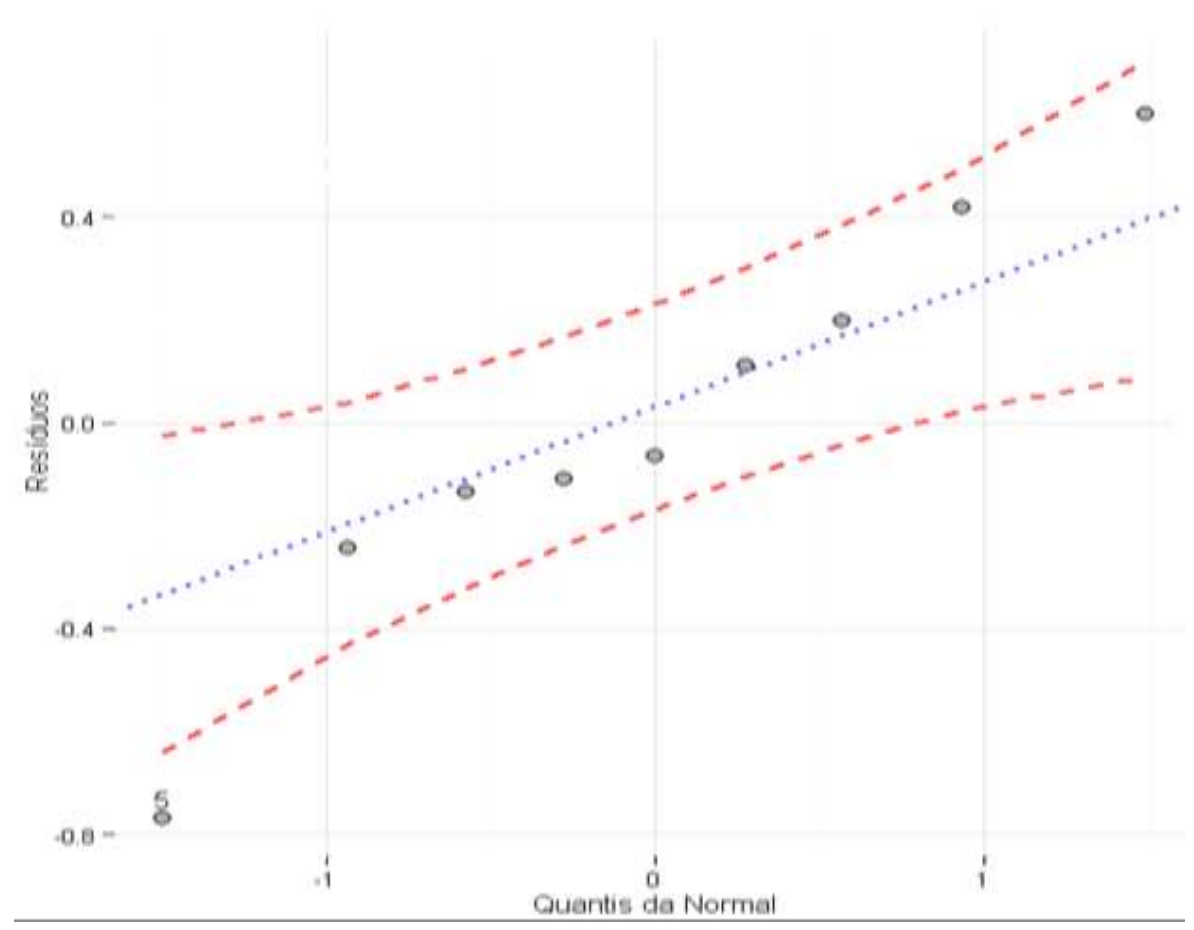

Fonte: Autores (2020).

Em relação a homocedasticidade utilizou-se o teste de Breusch Pagan (p-valor = 0,27) e para independência o teste de Durbin-Watson (p-valor =0,99). Sendo assim, os pressupostos de normalidade, homocedasticidade e independência são atendidos pelo Modelo Cúbico.

Para a análise de previsão do número de casos de esporotricose no município de Timbaúba-PE, utilizamos as médias móveis de comprimentos 3, 4, 5 e 7 de casos confirmados (Tabela 3). 
Tabela 3. Médias Móveis de Comprimentos (3-4-5-7).

\begin{tabular}{|c|c|c|c|c|c|}
\hline Mês & Teste positivo & MM 3 & MM 4 & MM 5 & MM 7 \\
\hline Janeiro & 2 & - & - & - & - \\
\hline Fevereiro & 6 & 3,67 & 3,5 & - & - \\
\hline Março & 3 & 4 & 3,25 & 3 & - \\
\hline Abril & 3 & 2,33 & 2,5 & 3,2 & 2,86 \\
\hline Maio & 1 & 2,33 & 2,25 & 2,4 & 2,71 \\
\hline Junho & 3 & 2 & 1,75 & 2 & 3 \\
\hline Julho & 2 & 2 & 3,5 & 3 & 2,86 \\
\hline Agosto & 1 & 3,67 & 3,25 & 3,2 & 3,14 \\
\hline Setembro & 8 & 3,67 & 4 & 3,6 & 3,14 \\
\hline Outubro & 2 & 5 & 4 & 3,4 & - \\
\hline Novembro & 5 & 2,67 & - & - & - \\
\hline Dezembro & 1 & - & - & - & - \\
\hline
\end{tabular}

Fonte: Autores (2020).

Segundo o Teste KPSS (Kwiatkowski-Phillips-Schmidt-Shin) a série temporal de casos confirmados de Esporotricose em Timbaúba é estacionária (p-valor $=0,1)$. Este teste é mais adequado para análise da série que o Dick-fuller, Dick-fuller aumentado e Phillips-Perron, por ter tamanho reduzido, ou seja, menor que 100 pontos (Margarido \& de Medeiros Júnior, 2006). Devido à série ser estacionária, pode-se realizar o modelo de médias móveis simples (Morettin \& Toloi, 2006). Sabe-se também que o modelo de médias móveis é aplicável a séries temporais de tamanho pequeno (Morettin \& Toloi, 2006).

Realizou-se em seguida os testes não paramétricos para análise da tendência dos casos positivos, os testes de WaldWolfwitz (p-valor =0,933), Cox-Stuart (p-valor =1,000) e Mann-Kendall (p-valor = 0,573), que por sua vez todos os métodos confirmam a hipótese de que a série de casos de Esporotricose não possui tendência.

Na Figura 4, pode-se observar o gráfico das médias móveis de comprimento 3, 4, 5 e 7 para o período de janeiro a dezembro de 2020. Percebe-se que a média móvel do comprimento 7 representa melhor o comportamento de longo prazo da série de casos confirmados de Esporotricose, por ter um comprimento maior, indicando uma ligeira oscilação em torno de 3 casos confirmados de Esporotricose em Timbaúba.

Figura 4. Médias Móveis (MM) dos casos confirmados de Esporotricose no município de Timbaúba em 2020.

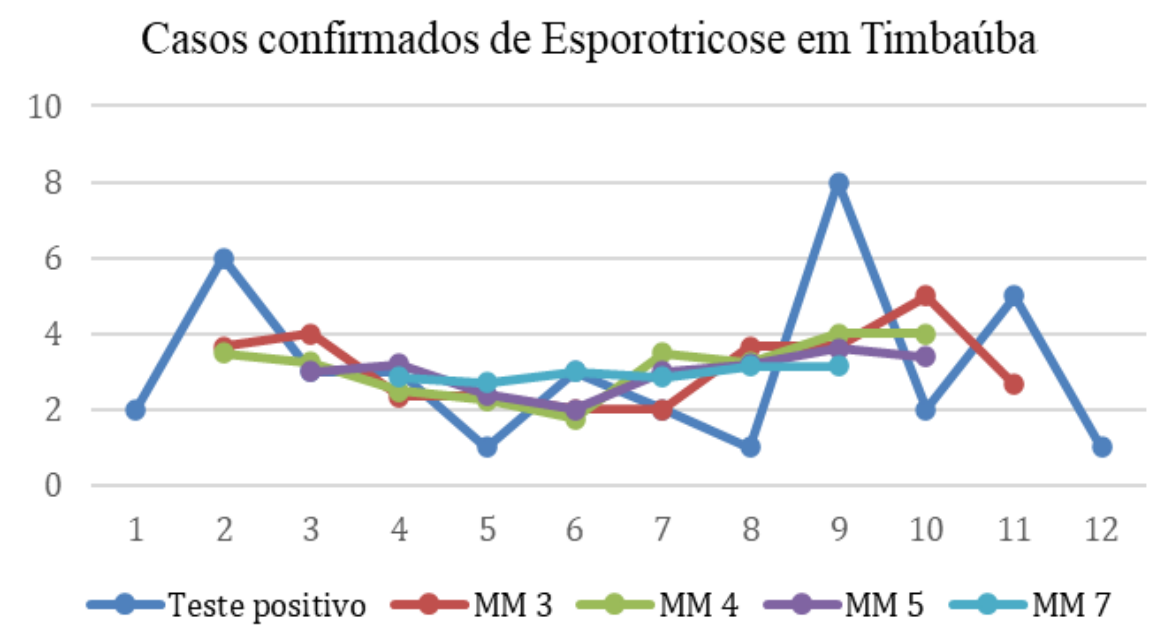

Fonte: Autores (2020)

Foi estimado o modelo de Média Móveis Simples (MMS) para o período de janeiro a dezembro de 2020 para do número de casos confirmados de esporotricose. As medidas de acurácia obtidas para o comprimento 3 foram de 1,37 para o 
Desvio Padrão Absoluto da Média (MAD) e de 3,22 para o Desvio Padrão Quadrático da Média (MSD). Em seguida fez-se a previsão do número de casos de Esporotricose para janeiro de 2021 através desse modelo. Desta forma, em janeiro de 2021 ocorrerão 2,66 IC $(0,98 ; 4,36)$ casos de esporotricose em Timbaúba, em um intervalo de confiança de $95 \%$ de acordo com a Tabela 4.

Já para comprimento 4, as medidas de acurácia obtidas foram MAD de 1,75, e o MSD de 4,75. Esse modelo prevê que em janeiro de 2021 ocorrerão 4 IC $(2,22 ; 5,78)$ casos de esporotricose em Timbaúba, em um intervalo de confiança de $95 \%$. Para comprimento 5 e 7 têm-se MAD 1,94 e 2,49 respectivamente, e MSD de 5,62 e 7,49 respectivamente. As previsões desses modelos para janeiro de 2021 podem ser observadas na Tabela 4. De acordo com os critérios MAD e MSD, o melhor modelo é o de médias móveis de comprimento da média 3.

Tabela 4. Critérios de decisão e previsão para o número e casos de Esporotricose no município de Timbaúba em janeiro de 2021.

\begin{tabular}{cccc}
\hline MMS & MAD & MSD & Previsão IC (95\%) \\
\hline Comprimento 3 & 1,37 & 3,22 & $2,66(0,98 ; 4,36)$ \\
Comprimento 4 & 1,75 & 4,75 & $4(2,22 ; 5,78)$ \\
Comprimento 5 & 1,94 & 5,62 & $3,4(1,66 ; 5,14)$ \\
Comprimento 7 & 2,49 & 7,49 & $3,14(1,45 ; 4,84)$ \\
\hline
\end{tabular}

Fonte: Autores (2020).

A representação gráfica da previsão de casos de esporotricose pelo modelo de suavização média móvel simples de comprimento 3 pode ser observada na Figura 5.

Figura 5. Modelo de Médias Móveis Simples, comprimento da média 3 e previsão para o número de casos confirmados de Esporotricose no município de Timbaúba - PE, 2020.

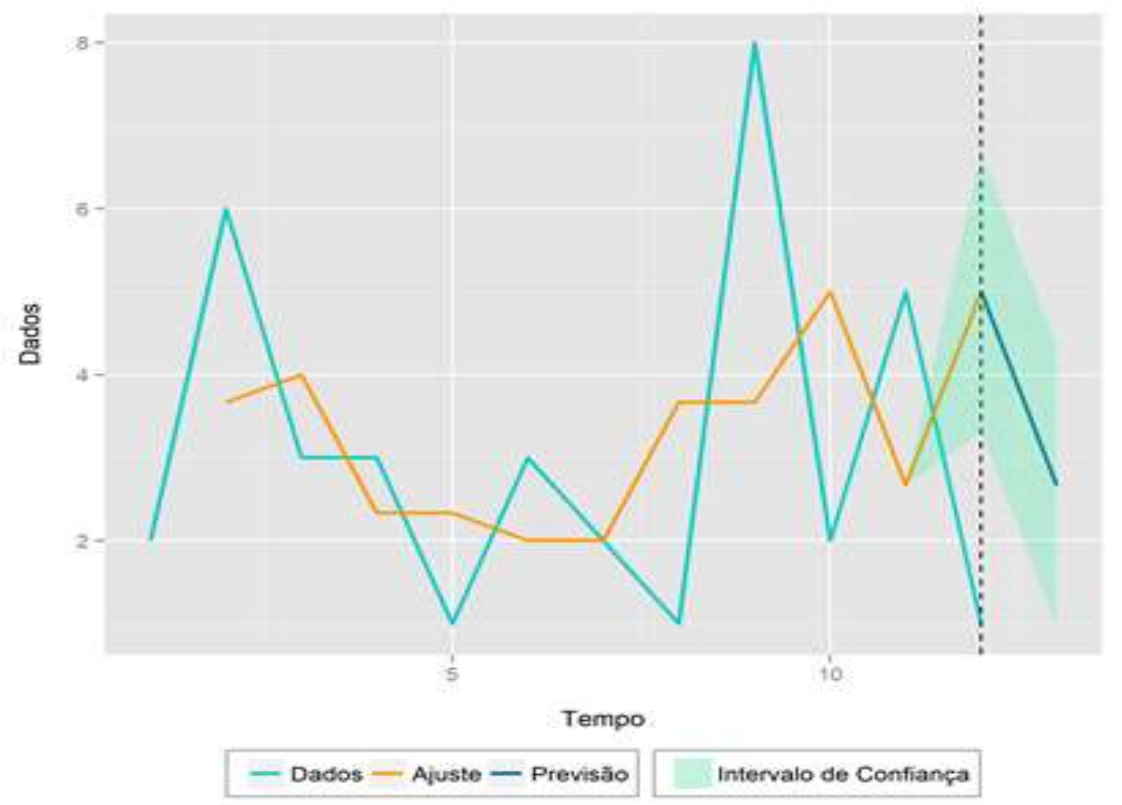

Fonte: Autores (2020). 


\subsection{Discussão de Diagnóstico e Tratamento}

Uma das técnicas de coleta empregada para a obtenção de material para a análise citológica foi a Biópsia por Agulha Fina (BAF) descrito seguramente com que expressa maior êxito para obtenção de material em lesões proliferativas.

O material também pode ser obtido por outras técnicas como por exemplo a técnica de impressão (Figura 6), onde uma lâmina de vidro para microscopia é posta em contato com a lesão ulcerada e o material é depositado na mesma, embora com frequência esse tipo de coleta não seja tão viável para a avaliação de neoplasias, pois pode apresentar apenas células inflamatórias na avaliação de células tumorais para a detecção de bactérias e fungos é uma boa opção (Cowell, Tyler, Meinkoth \& DeNicola, 2009).

Figura 6. Coleta de material de ferida ulcerada pela técnica de impressão.

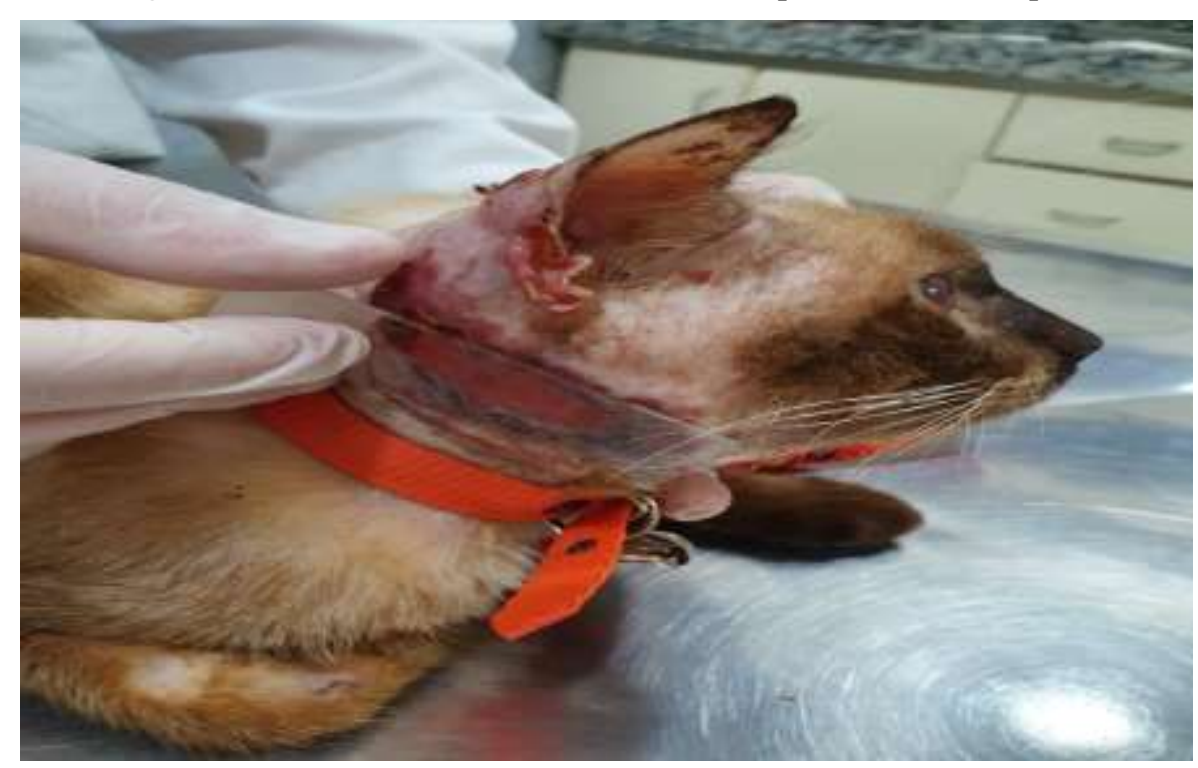

Fonte: Centro Veterinário Santa Cândida (2020).

Na Figura 6 observa-se a técnica de coleta de material com uma lâmina de vidro para microscopia em que a contenção e disposição do animal é de fundamental importância durante o processo de impressão do material a ser examinado.

Com o material coletado, e seco ao ar, deve ser fixado e corado (Guerra, Araújo, Ressio \& Fernandes, 2018). Os corantes tipo Romanowsky são bastante utilizados na rotina por diferentes vantagens, algumas delas são: baixo custo e praticidade. Embora a visualização dos detalhes do núcleo e nucléolo das células não seja tão evidente quando comparados com a utilização dos corantes. Para a utilização dos corantes tipo Romanowsky (Figura 7) as amostras secas ao ar ajudam no processo de adesão da célula na lâmina, impedindo que as células se percam durante o processo de coloração das mesmas (Cowell, Tyler, Meinkoth \& DeNicola, 2009). 
Figura 7. Fotomicrografia de lâmina de animal positivo para esporotricose onde foi utilizado corante tipo Romanowsky.

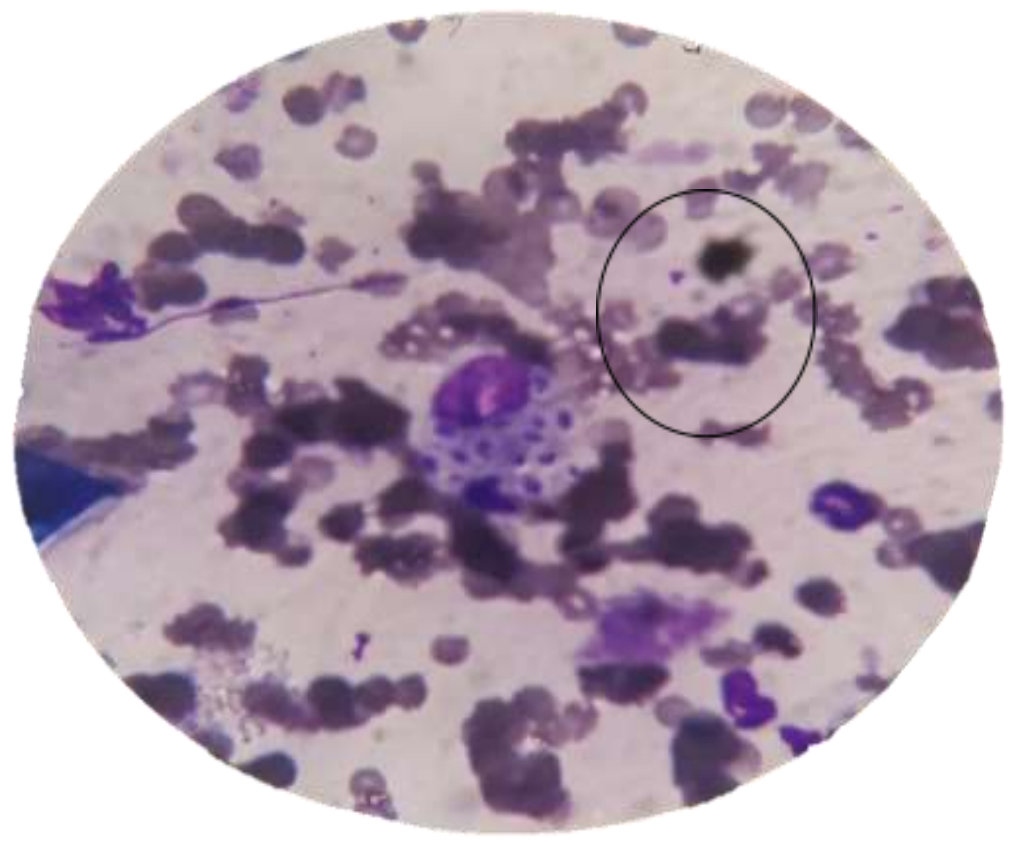

Fonte: Centro Veterinário Santa Cândida (2020)

Observamos na Figura 7 o resultado positivo para esporotricose com a coloração do material coletado pela técnica de impressão em lâmina de vidro utilizando o corante do tipo Romanowsky. Na Figura 8 é possível visualizar características de células leveduriformes correspondentes ao complexo Sporothrix scenckii.

Figura 8. Células leveduriformes características de agentes do complexo Sporothrix schenckii.

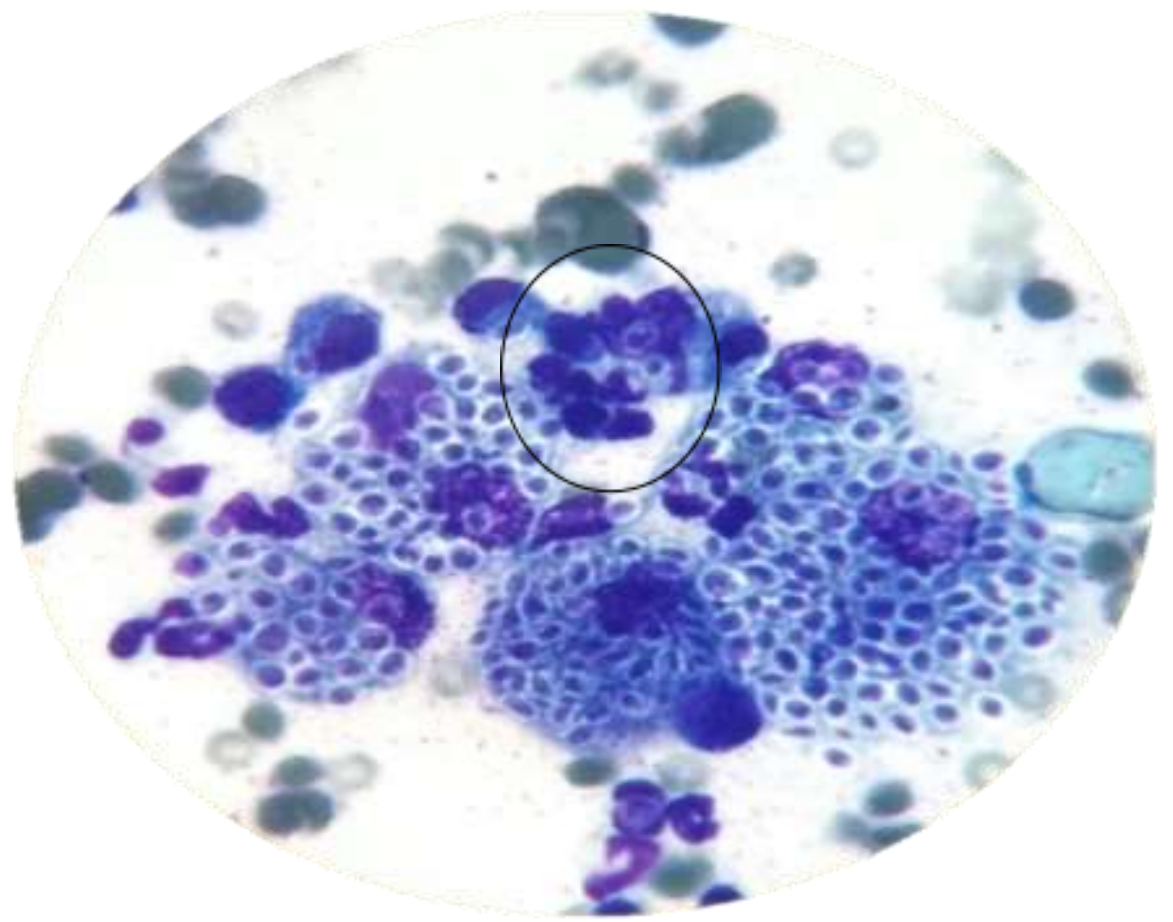

Fonte: Centro Veterinário Santa Cândida (2020) 
Por si tratar de fungo dimórfico, tem capacidade de transmissibilidade pelas formas micélio e levedura, tendo as células leveduriformes do complexo de Sporothrix schenckii como uma das mais comuns em infecção entre felinos (Santos et al., 2018).

Exemplificando um caso de tratamento, temos que Jack é um felino de aproximadamente 3 anos, pesando 3,550 quilogramas que foi atendido com histórico de que o animal apresentava ferida ulcerada a um pouco mais de um mês, tutora relata que tentou utilizar várias medicações por conta própria a fim de curar o animal dos ferimentos, todos se mostraram ineficazes.

Foi feito uma lâmina pela técnica de impressão da ferida e foi constatado a presença de leveduras do complexo Sporothrix schenckii, com esse achado foi prescrito para uso via oral silimarina $100 \mathrm{mg}$ a ser administrado com o animal em jejum para melhor absorção e itraconazol 100mg/animal a ser administrado junto ao alimento, ambos para serem administrado a cada 24 horas, e para uso tópico pomada a base de cetoconazol.

Quanto a terapêutica Crivellenti \& Borin-Crivellenti (2015), bem como Santos et al., (2018) citam que 05-10mg/kg de itraconazol a cada 12 ou 24 horas, Papich (2012) registra que pode ser utilizado 5mg/kg ou de 25 a $50 \mathrm{mg} / \mathrm{gato}$ e Nelson \& Couto (2015) afirmam que em gatos pode ser utilizado $100 \mathrm{mg} / \mathrm{gato} / \mathrm{dia}$ e esta foi a dosagem utilizada para o tratamento do animal supracitado nos dois primeiros meses de tratamento enquanto havia feridas ulceradas pelo corpo, após isso a dose foi corrigida para $10 \mathrm{mg} / \mathrm{kg} / \mathrm{dia}$. Como o itraconazol em doses elevadas pode causar hepatotoxidade foi prescrito a silimarina que é um hepatoprotetor utilizado para o tratamento de hepatopatias incluindo reações hepatotóxicas, Papich (2012) recomenda a dose de 5 a $15 \mathrm{mg} / \mathrm{kg}$ embora ressalte que para alguns autores são doses de no mínimo $30 \mathrm{mg} / \mathrm{kg}$. Assim como Nelson \& Couto (2015) recomendam de 50 a $200 \mathrm{mg} / \mathrm{cão} / \mathrm{dia}$.

O animal foi acompanhado por um período de quatro meses com o tratamento, a melhora clínica tornou-se evidente logo após o primeiro mês de tratamento, no segundo mês a remissão significativa da ferida. O animal foi acompanhado em visitas a cada 30 dias para reavaliação e acompanhamento (Figura 9). Assim como aconselham Crivellenti \& Borin-Crivellenti (2015) e Santos et al., (2018) o tratamento perdura até 30 dias depois da remissão completa da feria a fim de evitar recidiva da doença.

Figura 9. A. Lesão ulcerada em felino na região periauricular; B. Evolução da lesão após $1^{\circ}$ mês de tratamento; C. Lesão após $2^{\circ}$ mês de tratamento.
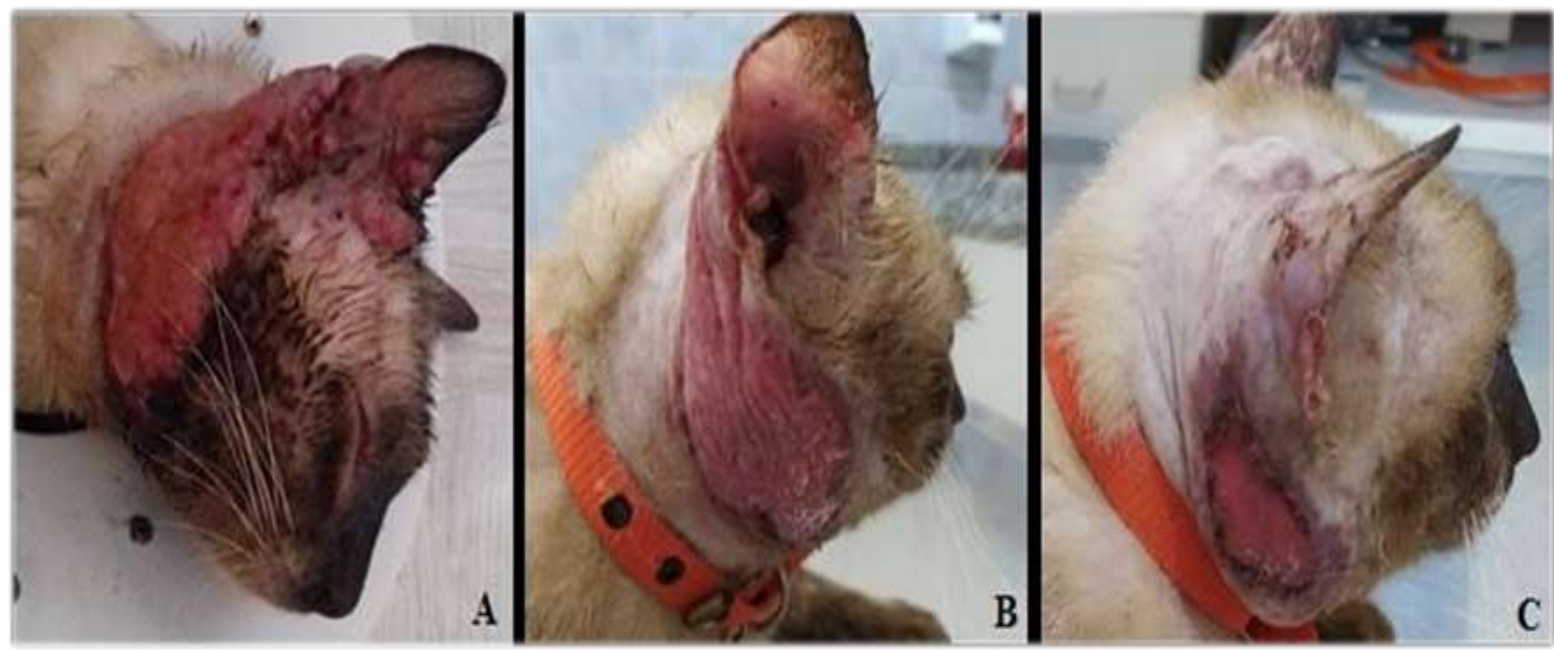

Fonte: Centro Veterinário Santa Cândida (2020). 
Na Figura 9 pode-se observar que a lesão ulcerada tende a reduzir até cicatrização, desde que as recomendações médicas sejam seguidas rigorosamente pelo tutor, com acompanhamento do profissional médico veterinário. Após o período de tratamento o animal ficou recuperado (Figura.10).

Figura 10. Animal após tratamento, sem apresentar as lesões ulceradas.
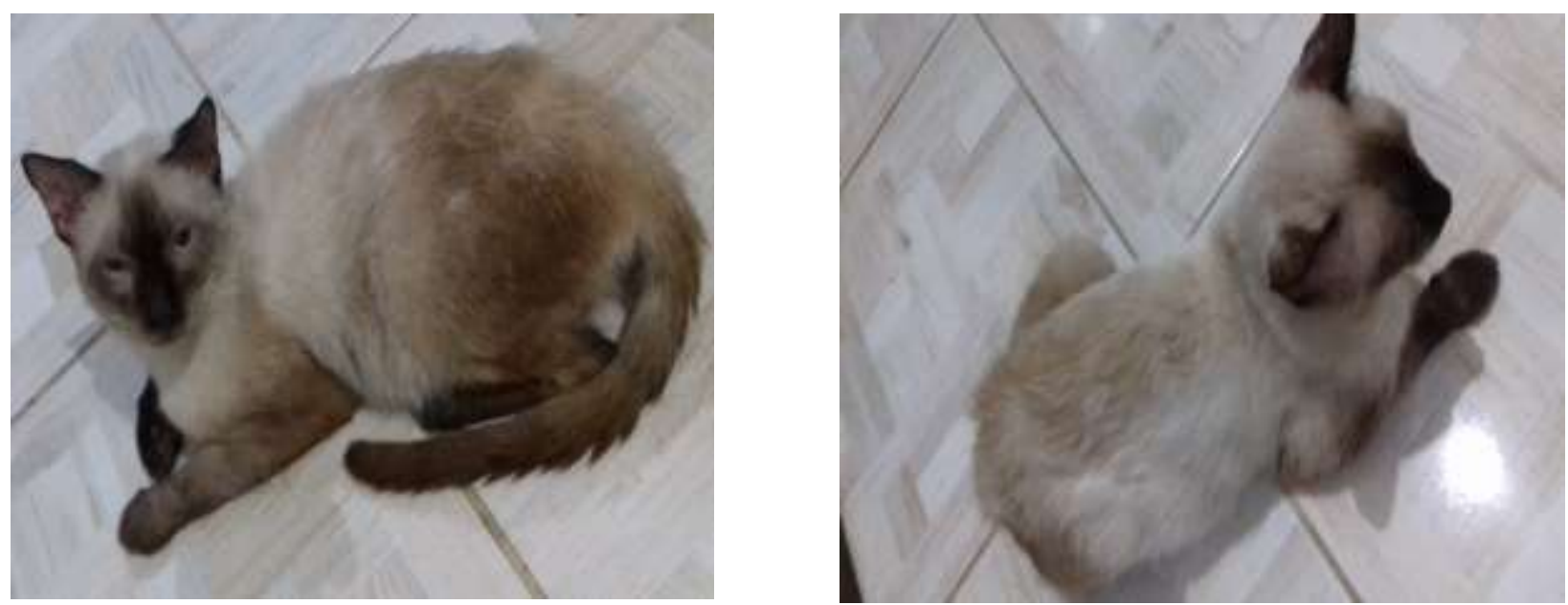

Fonte: Centro Veterinário Santa Cândida (2020).

É possível verificar na Figura 10 que todas as lesões ulceradas foram cicatrizadas e, desta forma o animal Jack recebeu alta do tratamento.

\section{Considerações Finais}

O Modelo de Regressão Cúbico se mostrou como melhor modelo ajustado aos dados, uma vez que atendeu todos os pressupostos de normalidade, homocedasticidade e independência. Pois, obteve o melhor valor para o Coeficiente de Determinação $\left(R^{2}\right)$ em relação aos demais modelos utilizados.

Desta forma, pode-se concluir que os casos de Esporotricose no município de Timbaúba oscilam em torno de 3 casos confirmados, e que não possuiu tendência de mudança na ocorrência dessa doença no município de Timbaúba em 2020. Para o mês de janeiro de 2021, obtivemos uma previsão de 2,66 IC $(0,98 ; 4,36)$ casos de esporotricose em Timbaúba, em um intervalo de confiança de 95\% através do modelo de média móveis simples, comprimento da média 3.

Quanto ao tratamento e cuidados com os animais infectados, a exemplificação com o animal Jack mostrou eficiência do tratamento e acompanhamento clínico. Contudo, não houve evidências de que os procedimentos de tratamentos são efetivamente conduzidos pelos demais tutores, uma vez que não se obteve registros do retorno destes animais durante e pós tratamento.

Sendo assim, faz-se necessário estudos futuros mais aprofundados sobre a evolução da doença com resultados esclarecedores que apontem para a erradicação, ou não, da doença no município de Timbaúba, bem como demais localidades em que haja evidências da esporotricose no estado pernambucano.

\section{Agradecimentos}

À Universidade Federal Rural de Pernambuco - UFRPE; ao Programa de Pós-Graduação em Biometria e Estatística Aplicada - PPGBEA; à Coordenação de Aperfeiçoamento de Pessoal de Nível Superior - CAPES; ao Conselho Nacional de Desenvolvimento Científico e Tecnológico - CNPq. e ao Centro Veterinário Santa Cândida - Timbaúba/PE - Brasil. 


\section{Referências}

Araujo, A. K. L., \& Leal, C. A. S. (2016). Esporotricose felina no município de Bezerros, Agreste Pernambucano: Relato de caso. Pubvet, 10(11), 816-820. doi: https://doi.org/10.22256/pubvet.v10n11.816-820

Bittencourt, M. S., Weber, A. P., Portella, J. E., Cardoso, N. Q., dos Santos, C. C. P., \& dos Santos Monti, F. (2016). Esporotricose Felina Refratária à Terapia-Relato de Caso. Revista eletrônica biociências, biotecnologia e saúde, 6(15), 128-130.

Camelo, H. D. N., Lucio, P. S., Leal Junior, J. B. V., \& de Carvalho, P. C. M. (2017). Métodos de previsão de séries temporais e modelagem híbrida ambos aplicados em médias mensais de velocidade do vento para regiões do Nordeste do Brasil. Revista Brasileira de Meteorologia,32(4), 565-574. https://doi.org/10.1590/0102-7786324005

Canhanga, P. E. (2020). Modelo matemático para previsão de vendas: Regressão Linear Simples. Cadernos do IME-Série Matemática, (14), 71-81.

Couto, H. J. B., Oliveira, R. A. E., \& Braga, P. F. A. (2016). Previsão de Radiação Solar Incidente no Estado do Ceará-Brasil. Holos, 7, 218-230. https:// 10.15628/holos.2016.2706

Cowell, R. L., Tyler, R. D., Meinkoth, J. H., \& DeNicola, D. B. (2009). Diagnóstico citológico e hematologia de cães e gatos. Medvet, (3a ed.), 476p.

Crivellenti, L. Z., \& Borin-Crivellenti, S. (2015). Casos de rotina em medicina veterinária de pequenos animais. MedVet, (2a ed.), 842p.

de Campos, M. I., \& Rueda, F. J. (2017). Regressão linear e quadrática: Análises comparativas de efeito em medidas do comportamento organizacional. Estudos de Psicologia, 22(2), 225-234.

de Macêdo-Sales, P. A., da Silveira Souto, S. R. L., Destefani, C. A., de Lucena, R. P., da Rocha, E. M. D. S., \& de Souza Baptista, A. R. (2018). Diagnóstico laboratorial da esporotricose felina em amostras coletadas no estado do Rio de Janeiro, Brasil: limitações da citopatologia por imprint. Revista Pan-Amazônica de Saúde, 9(2), 13-19.

de Souza, E. W., Borba, C. de M., Pereira, S. A., Gremião, I. D. F., Langohr, I. M., Oliveira, M. M. E., de Oliveira, R. de V. C., da Cunha, C. R., ZancopéOliveira, R. M., de Miranda, L. H. M., \& de Souza, R. C. M. (2018). Clinical features, fungal load, coinfections, histological skin changes, and itraconazole treatment response of cats with sporotrichosis caused by Sporothrix brasiliensis. Scientific Reports, 8(1), 1-10. https://doi.org/10.1038/s41598-018-27447-5

do Amaral, L. S., dos Santos, A. L. P., de Figueiredo, M. P. S., de Almeida Ferreira, D. S., Silva, J. E., dos Santos, H. C. T., \& Moreira, G. R. (2020). Interiorização do Covid-19: Uma análise da evolução dos casos/10 mil habitantes em municípios da Microrregião de Garanhuns no Estado de Pernambuco, através de modelos de Regressão não linear. Research, Society and Development, 9(9), e293996582-e293996582. http://dx.doi.org/10.33448/rsd-v9i9.6582

dos Santos, A. L. P., de Figueiredo, M. P. S., Ferreira, T. A. E., Gomes-Silva, F., Moreira, G. R., Silva, J. E., \& de Freitas, J. R. (2020). Análise e previsão da evolução do número de óbitos por COVID-19 do estado de Pernambuco e Ceará utilizando modelos de regressão. Research, Society and Development, 9(7), e602974551-e602974551. http://dx.doi.org/10.33448/rsd-v9i7.4551

Equipe Estatcamp (2014). Software Action. Estatcamp- Consultoria em estatística e qualidade, São Carlos - SP, Brasil. URL http://www.portalaction.combr/.

Guerra, J. M., Araújo, L. J. T. D., Ressio, R. A., \& Fernandes, N. C. C. D. A. (2018). Patologia morfológica e molecular aplicadas à inovação diagnóstica e vigilância da leishmaniose visceral. Revista do Instituto Adolfo Lutz, e1762-e1762, 1-7.

Margarido, M. A., \& de Medeiros Junior, H. (2006). Teste para mais de uma raiz unitária: uso do software SAS® na elaboração de uma rotina para o teste dickey-pantula. Pesquisa \& Debate. Revista do Programa de Estudos Pós-Graduados em Economia Política, 17(1 (29)).

Mascarenhas, M. B., Botelho, C. B., Manier, B. S. M. L., Costa, T. S., \& Fernandes. J. I. (2019). An unusual case of feline otitis externa due to sporotrichosis. Journal of Feline Medicine and Surgery Open Reports, 5(1), 2055116919840810. http:// 10.1177/2055116919840810

Morettin, P. A., \& Toloi, C. Análise de séries temporais. (2a ed.), Editora Edgard Blucher.

Nelson, R., \& Couto, C. G. (2015). Medicina interna de pequenos animais. Elsevier Brasil.

Papich, M. G. (2012). Manual Saunders de Terapia Veterinária. Elsevier Health Sciences Brazil. 858p.

Pereira, A. S., Shitsuka, D. M., Parreira, F. J., \& Shitsuka, R. (2018). Metodologia da pesquisa científica. Ed. UAB/NTE/UFSM. https://repositorio.ufsm.br/bitstream/handle/1/15824/Lic_Computacao_Metodologia-Pesquisa-Cientifica.pdf.

Portal Action. Médias Móveis Simples. http://www.portalaction.com.br/series-temporais/31-medias-moveis-simples-mms.

Rios, M. E., Suarez, J., Moreno, J., Vallee, J., \& Moreno, J. P. (2018). Zoonotic Sporotrichosis Related to Cat Contact: First Case Report from Panama in Central America. Cureus, 10(7), e2906. http://10.7759/cureus.2906

Santos, A. F., Rocha, B. D., Bastos, C. V., Oliveira, C. S. F., Soares, D. F. M., Pais, G. C. T., \& Brandão, S. T. (2018). Guia prático para enfrentamento da esporotricose felina em Minas Gerais. Revista Veterinária \& Zootecnia em Minas, 137(38), 16-27.

Silva, J. E., Santos, A. L. P., Freitas, J. R. de, Cunha, A. L. X., Shinohara, N. K. S., \& Cunha Filho, M. (2020a). Study of sporotrichosis at the national and international level with a statistical focus: a systematic review of zoonosis. Research, Society and Development, 9(11), e83591110461. https://doi.org/10.33448/rsd-v9i11.10461

Silva, M. M. de L., Cordeiro, N. M., Nascimento, G. I. L. A., Amaral, L. S. do, Jesus, E. S., Rocha, J. S., Santos, H. C. T., Silva, J. E., Silva, W. R. L., Silva, T. C. de M., Silva, R. P., Guimarães, J. F. A., Silva, J. A. A., Moreira, G. R., Cunha Filho, M., \& Silva, A. S. A. (2020b). Comparative study of sugar cane production in the microregion of Vitória de Santo Antão/PE, Brazil. Research, Society and Development, 9(11), e2669119857. https://doi.org/10.33448/rsdv9i11.9857 
Research, Society and Development, v. 10, n. 1, e51310112082, 2021

(CC BY 4.0) | ISSN 2525-3409 | DOI: http://dx.doi.org/10.33448/rsd-v10i1.12082

Sizar, O., \& Talati, R. (2019). Sporotrichosis (Sporothrix Schenckii). In: StatPearls [Internet]. Treasure Island (FL): StatPearls Publishing. https://www.ncbi.nlm.nih.gov/books/NBK532255/.

Souza, A. A. A. (2016). Comparação entre modelo de regressão e séries temporais para a arrecadação do IPVA em Sergipe. Monografia de Curso de Bacharel em Administração pela Universidade Federal de Sergipe. 